\title{
Characterization of Blood Flow Changes in Normal and Pathological Aortic Dilation from 4D Flow Magnetic Resonance Imaging
}

\author{
Sophia Houriez--Gombaud-Saintonge ${ }^{1,2}$, Ariel Pascaner ${ }^{4}$, Gilles Soulat ${ }^{3}$, Umit Gencer $^{3}$, Thomas $^{3}$ \\ Dietenbeck $^{1}$, Damian Craiem ${ }^{4}$, Emilie Bollache ${ }^{1}$, Yasmina Chenoune ${ }^{2}$, Elie Mousseaux ${ }^{3}$, Nadjia \\ Kachenoura $^{1}$ \\ ${ }^{1}$ Sorbonne Université, INSERM, CNRS, Laboratoire d'Imagerie Biomédicale (LIB), Paris, France \\ ${ }^{2}$ ESME Sudria Research Lab, Paris, France \\ ${ }^{3}$ Hopital Européen Georges Pompidou, Paris, France \\ ${ }^{4}$ IMETTYB - Universidad Favaloro - CONICET, Buenos Aires, Argentina
}

\begin{abstract}
Aim: Maximal diameter (Dmax), which is commonly used to diagnose thoracic aortic aneurysm (TAA) was previously shown to be normal in 20-30\% of patients who ultimately develop dissection. Besides, inner aortic flow is associated with its wall dynamics. Thus, our aim was to quantify aortic flow changes using $4 D$ flow MRI in the setting of ascending aorta (AA) dilation. Methods: We studied 20 patients with TAA and tricuspid aortic valve (TAA) and 56 healthy controls (30 subjects, $36 \pm 9 y \leq 50$ years named $Y C, 26$ subjects, $65 \pm 9 y>50$ years named OC). All underwent $4 D$ flow MRI. After aortic segmentation, regional volume of backward flow $\left(V_{B F}\right)$ was extracted in addition to in-cross-section velocity standard deviation (SD) as well as maximal velocity jet angle (Angle) and eccentricity (Ecc). Receiver operating characteristic (ROC) analysis was performed to assess ability of flow indices to characterize dilation. Results: While AA Dmax changed by 1.4 folds between TAA and $O C, V_{B F}$ changed by 6.5 folds, and Ecc, Angle and SD changed by 1.3 to 1.9 folds between the two groups. Moreover $V_{B F}$ varied consistently with age and was able to detect $A A$ dilation with an accuracy of 0.98 . Conclusion: $4 D$ flow MRI indices of local aortic flow disorganization, specifically backward flow, were able to accurately characterize dilation.
\end{abstract}

\section{Introduction}

While it is well-known that the ascending aorta (AA) dilates with aging, dilation above a predefined maximal diameter threshold is treated to prevent aortic dissection [1]. However with the use of such geometric criterion alone, around $30 \%$ of individuals with aortic size below the surgical threshold end up with aortic dissection [2, 3]. This highlights the need of new imaging biomarkers that would be less sensitive to body size and altered at the early stage of the disease. 3D time-resolved flow magnetic resonance imaging (4D flow MRI) provides both volumetric anatomy and blood flow velocities in all space directions during the entire cardiac cycle, and thus a comprehensive visualization and flow quantification. Several studies $[4,5]$ demonstrated the usefulness of 4D flow MRI-derived indices in selected groups of patients with AA dilation and with either a tricuspid or a bicuspid aortic valve. Thus, our objectives were to: 1) quantitatively characterize local flow changes along the aorta from 4D flow MRI, and 2) test the relevance of the derived flow indices in aging as well as with the presence of aortic root dilation with a tricuspid aortic valve.

\section{Material and methods}

\subsection{Population and data acquisition}

We retrospectively included 56 healthy subjects (mean age: $49 \pm 17$ years, 32 women), without cardiovascular disease as well as 20 patients $(65 \pm 14$ years, 7 women) with aortic root dilation and tricuspid aortic valve (TAA). TAA patients had no severe aortic insufficiency and no prior aortic surgery. All participants of this study gave their informed consent and study protocol was approved by the local ethics committee. All subjects underwent an MRI exam on a 3T magnet (GE Healthcare). 4D flow data were acquired during free breathing with prospective ECG gating in a sagittal oblique volume encompassing the thoracic aorta with the following scan parameters: velocity encoding $=250 \mathrm{~cm} / \mathrm{s}$, voxel size $=1 \times 1.48 \times 2.38 \mathrm{~mm}^{3}$, echo time $=1.7 \mathrm{~ms}$, repetition time $=4.3-4.4 \mathrm{~ms}$ and flip angle $=15^{\circ}$. Data were reconstructed into 50 frames per cardiac cycle. All subjects also had an anatomical 3D spoiled gradient echo SPGR dataset acquired on a single temporal phase 

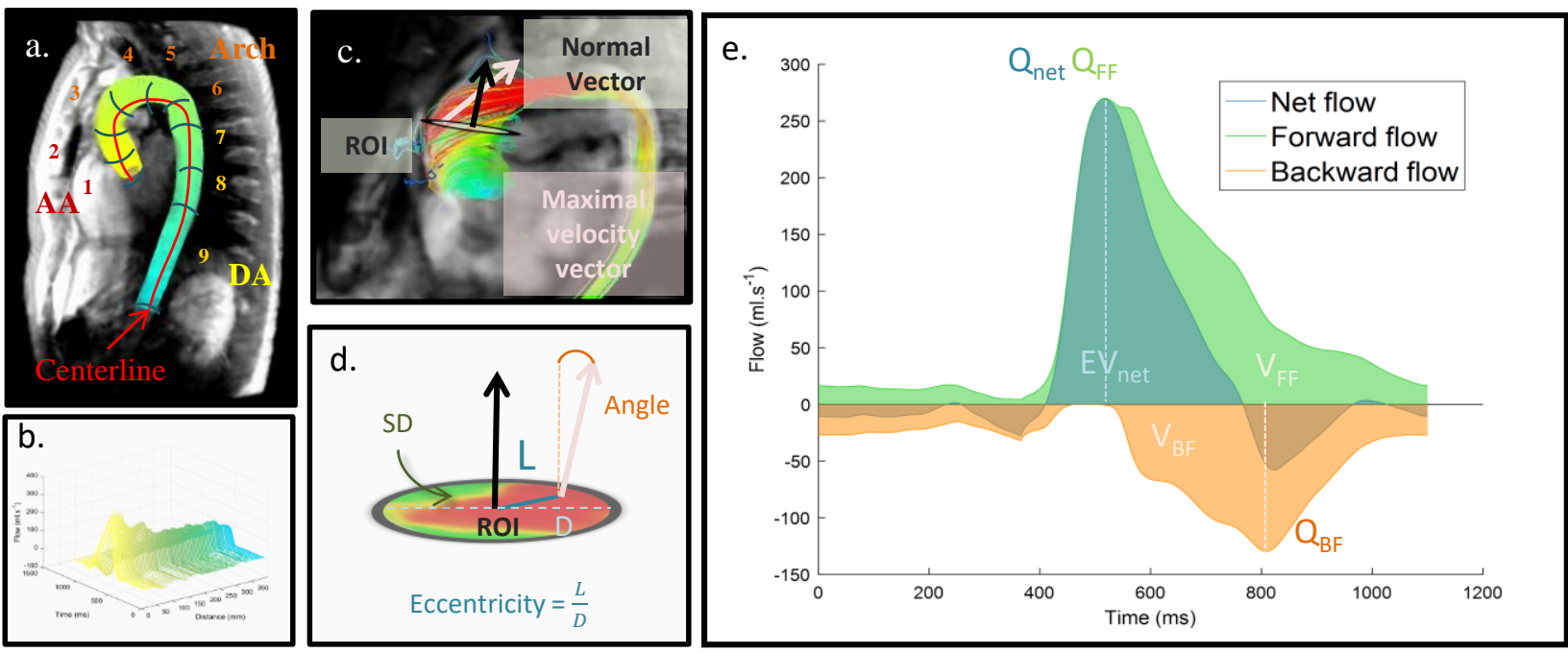

Figure 1. Methodological workflow: a) aortic segmentation, b) Net flow extracted from aortic segmentation on 4D flow MRI data along the centerline, c) 4D flow MRI systolic streamlines in a TAA patient and illustration of the vector normal to the cross-section and maximal velocity vector, d) flow angle, eccentricity and standard deviation (SD) extraction, e) time-resolved net, forward and backward flow curves and the corresponding peaks and volumes.

triggered during the diastolic phase with the following scan parameters: flip angle $=65^{\circ}$, repetition time $=3.6 \mathrm{~ms}$, echo time $=1.5 \mathrm{~ms}$, slice thickness $=3.6 \mathrm{~mm}$, pixel spacing $=0.78 \times 0.78 \mathrm{~mm}^{2}$ and spacing between slices $=0.9$ $\mathrm{mm}$.

\subsection{Aortic geometry}

SPGR data were used for a precise evaluation of aortic morphology using a semi-automated segmentation of the aorta (MIMOSA software [6]), after manually positioning 10 anatomical landmarks (Figure 1 a). Diameters were estimated perpendicular to the centerline along the aorta.

\subsection{Aortic blood flow functional indices}

4D flow MRI modulus images were used to manually delineate aortic borders on several consecutive crosssectional slices equally distributed perpendicular to the centerline along the whole aorta. Linear interpolation was then used to account for inter-slice gaps. This segmentation was superimposed on velocity data to estimate time-resolved net, forward and backward flows in all consecutive slices (Figure $1 \mathrm{a}-\mathrm{b}$ ).

Systolic peaks of forward and backward flow rate curves $\left(\mathrm{Q}_{\mathrm{FF}}, \mathrm{Q}_{\mathrm{BF}}, \mathrm{mL} / \mathrm{s}\right)$ and their volumes $\left(\mathrm{V}_{\mathrm{FF}}, \mathrm{V}_{\mathrm{BF}}\right.$, $\mathrm{mL}$ ), calculated as the area under curves, were extracted. Total ejection volume $\left(\mathrm{EV}_{\text {net }}, \mathrm{mL}\right)$ was calculated as the area under the net flow curve. Ratios of backward to forward flow peaks and volumes were then calculated as: $\mathrm{Q} \%=100 \times \frac{\mathrm{Q}_{\mathrm{BF}}}{\mathrm{Q}_{\mathrm{FF}}}$ and $\mathrm{V} \%=100 \times \frac{\mathrm{V}_{\mathrm{BF}}}{\mathrm{V}_{\mathrm{FF}}}$ (Figure. $\left.1 \mathrm{e}\right)$.
Moreover, the angle between the normal vector of each cross-section and the maximal velocity vector (Angle) was computed. Flow displacement (namely, eccentricityEcc) of the forward flow was also computed, as the distance between the cross-sectional center of mass and the maximal velocity, divided by the cross-section diameter [7]. Finally, in-slice standard deviation (SD) of velocities magnitude was computed for each cross-section (Figure. 1, c-d). For Angle, Ecc and STD only peak systole was considered in the remaining parts of the manuscript. Of note, the centerline resulting from $4 \mathrm{D}$ flow data was registered to the SPGR centerline using the Procrustes algorithm to allow comparison of the same aortic segments in terms of morphology and flow. Such segments corresponded to 1) the ascending aorta (AA), 2) the arch and 3) the descending aorta (DA) (Figure 1 a).

\subsection{Statistical analysis}

Our population was divided into 3 subgroups: 1) healthy controls $\leq 50$ years old, 2) healthy controls $>50$ years old and 3) TAA patients. Continuous variables are provided as medians and interquartile ranges. The twosided Wilcoxon rank sum test was used for comparisons between groups 1 and 2 as well as groups 2 and 3. To measure the ability of each parameter to distinguish between TAA patients and elderly subjects, receiver operating characteristic (ROC) curves analysis was used and the area under the curve (AUC), accuracy, sensibility, sensitivity as well as the abnormality thresholds using the Youden's index were provided. A p-value $<0.05$ indicated statistical significance. 


\section{Results and discussion}

The entire processing of a 4D flow MRI dataset including aortic segmentation took less than 10 minutes on a standard computer. Table 1 summarizes basic characteristics of the population. No significant differences were found for age and blood pressures between controls $>50$ years and TAA patients.

Table 1: Healthy controls and TAA basic characteristics $\left(\leq 50 \mathrm{y} v s .>50 \mathrm{y}:{ }^{* *} \mathrm{p}<0.01,{ }^{* * *} \mathrm{p}<0.001 \mid\right.$ TAA $v s .>50 \mathrm{y}$ :

\begin{tabular}{|c|c|c|c|}
\hline & $<50 y$ & $>50 y$ & TAA \\
\hline $\mathbf{N}$ & 30 & 26 & 20 \\
\hline $\begin{array}{c}\text { Gender } \\
\text { (female \%) }\end{array}$ & 60 & 54 & 33 \\
\hline $\begin{array}{c}\text { Age } \\
\text { (years) }\end{array}$ & $\begin{array}{c}35 \\
(28-43.7)\end{array}$ & $\begin{array}{c}67 * * * \\
(57-71.9)\end{array}$ & $\begin{array}{c}65 \\
(61-76.3)\end{array}$ \\
\hline $\begin{array}{c}\text { HR } \\
(\text { bpm) }\end{array}$ & $\begin{array}{c}66 \\
(62-75)\end{array}$ & $\begin{array}{c}64 \\
(59-68)\end{array}$ & $\begin{array}{c}60 \\
(57-73.5)\end{array}$ \\
\hline $\begin{array}{c}\text { SBP } \\
(\mathrm{mmHg})\end{array}$ & $\begin{array}{c}100 \\
(97-116)\end{array}$ & $\begin{array}{c}120 * * \\
(110-124)\end{array}$ & $\begin{array}{c}120 \\
(100-134)\end{array}$ \\
\hline $\begin{array}{c}\text { DBP } \\
(\mathrm{mmHg})\end{array}$ & $\begin{array}{c}76 \\
(68-82.5)\end{array}$ & $\begin{array}{c}78 \\
(73-84)\end{array}$ & $\begin{array}{c}77 \\
(74-87.4)\end{array}$ \\
\hline $\begin{array}{c}\text { BMI } \\
\left(\mathrm{kg} \cdot \mathrm{m}^{-2}\right)\end{array}$ & $\begin{array}{c}22 \\
(21-24.8)\end{array}$ & $\begin{array}{c}24 * * \\
(22-26.3)\end{array}$ & $\begin{array}{c}27^{+++} \\
(23-31.2)\end{array}$ \\
\hline $\begin{array}{c}\text { SPGR max diameter } \\
(\mathrm{mm})\end{array}$ & $\begin{array}{c}28 \\
(26-30.4)\end{array}$ & $\begin{array}{c}31 * * * \\
(30-32.5)\end{array}$ & $\begin{array}{c}43^{+++} \\
(40-46.8)\end{array}$ \\
\hline $\begin{array}{c}\mathrm{EV}_{\text {net }} \\
(\mathrm{ml})\end{array}$ & $\begin{array}{c}77 \\
(66-85)\end{array}$ & $\begin{array}{c}61^{* *} \\
(56-81)\end{array}$ & $\begin{array}{c}70 \\
(55-92)\end{array}$ \\
\hline
\end{tabular}

\subsection{Flow indices and their changes with age and aortic dilation}

Figure 2 summarizes flow indices over the 3 study groups and the 3 aortic segments (AA, arch and DA).

First, despite the expected and significant $(\mathrm{p}<0.01)$ drop in net flow volume with age, no significant differences were found between elderly subjects and TAA (Table 1), independent of the considered aortic segment. This finding highlights the adaptive remodeling process to sustain a preserved flow to peripheral organs despite the pronounced aortic root dilation. Such adaptive process might explain the lack of association between net flow and aortic diameter.

Second, while backward flow volume was only slightly predominant in the AA of healthy subjects as compared to the aortic arch and DA, it was increased on average by 12.7 folds in the AA of TAA patients as compared to their DA (Figure 2). Besides, backward flow volume was in average 6.5 higher in the AA of TAA patients than in elderly subjects. Interestingly, this increase was substantially higher than the change in maximal diameters between TAA and elderly controls, which was equal to 1.4 in average. The increasing ratio between TAA and elderly subjects in terms of backward flow volume dropped towards the DA. Such findings were also observed when considering backward to forward flow volume and peak ratios. Furthermore, in line with previous MRI findings, backward flow volume increased significantly with age.

Finally, indices of spatial blood flow organization, namely the angle, Ecc and velocity SD, were significantly increased (1.3-1.9 folds) in the AA of TAA patients, as compared to elderly controls.

\subsection{Ability of flow indices to characterize TAA patients}

Ability of AA flow indices to characterize TAA patients from elderly controls was summarized through ROC analysis results (Figure 3). Backward flow volume and peak indices outperformed all other indices as indicated by the high AUC and overall accuracy $(\geq 0.98)$. Indeed, the previously described indices such as Angle and Ecc were able to characterize TAA patients with an accuracy of 0.89 and 0.78 , respectively.
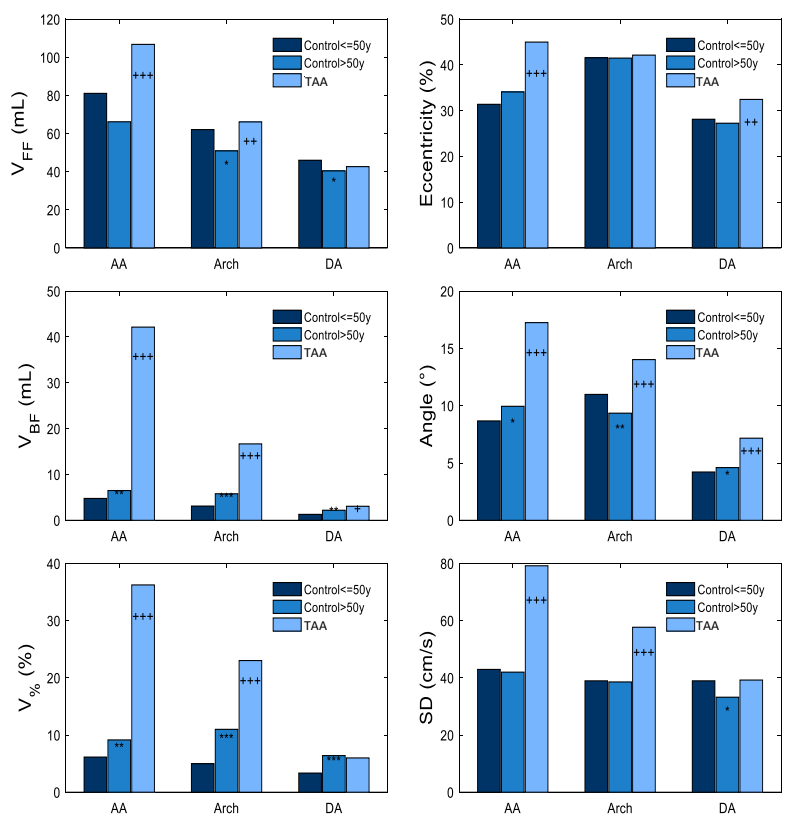

Figure 2. Distribution of median flow indices according to the study group and aortic segment. AA and DA: ascending and descending aorta, TAA: Aneurismal patients with tricuspid valve $(*: \mathrm{p}<0.05, * *: \mathrm{p}<0.01, * * *: \mathrm{p}<0.001$ for $\leq 50 \mathrm{y} v$ s. $>50 \mathrm{y} ;{ }^{+}: \mathrm{p}<0.05,{ }^{++}: \mathrm{p}<0.01,{ }^{+++}: \mathrm{p}<0.001$ for TAA vs. $>50 \mathrm{y})$.

\section{changes \\ A more local evaluation of flow}

Although this study was focused on segmental analysis, the developed workflow could be extended to a more localized assessment of flow disorganization in order to map "at risk area" either while using a single flow index or their combination to build-up a flow score, 

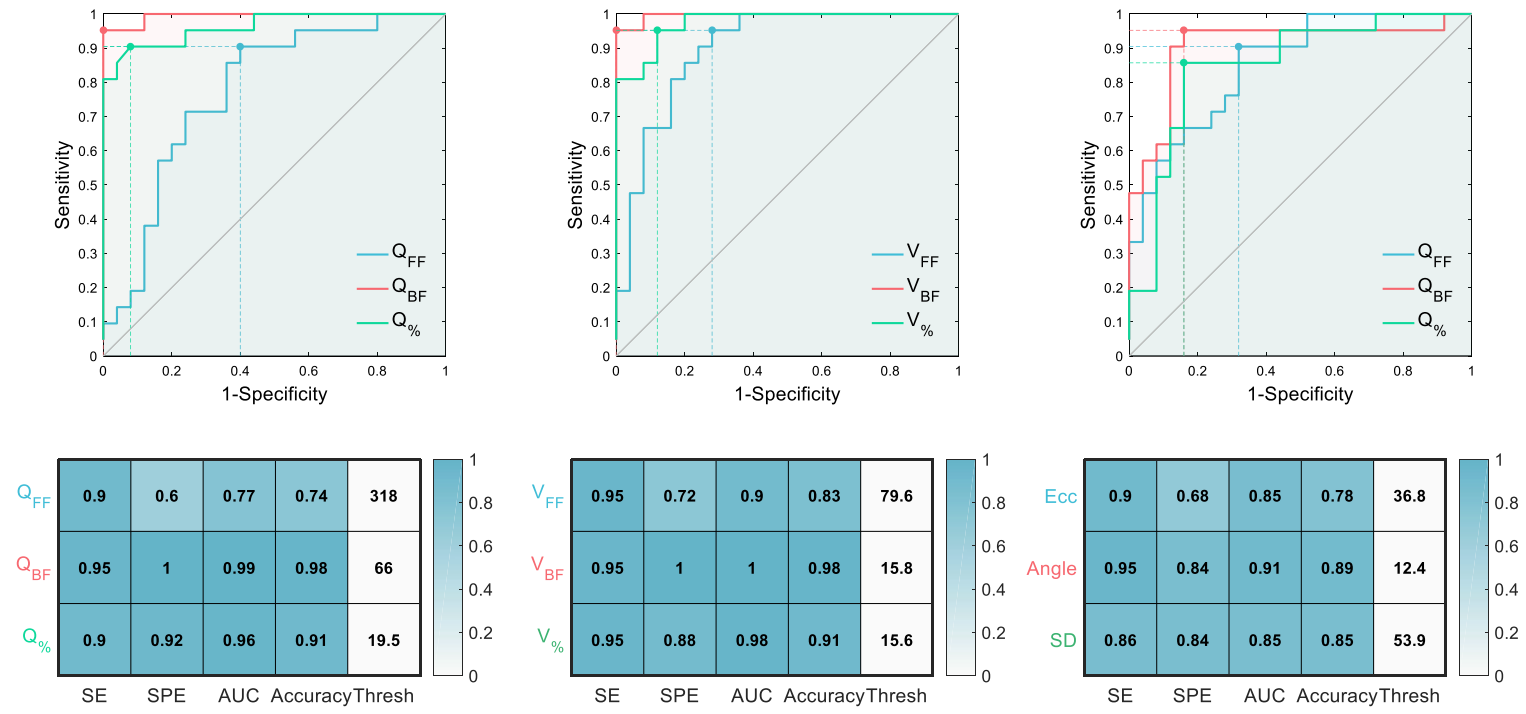

Figure 3. ROC analysis for AA flow indices. SE: sensitivity, SPE: specificity, AUC: area under the curve, Q: peak $(\mathrm{mL} / \mathrm{s})$ V: Volume $(\mathrm{mL})$, Ecc: eccentricity $(\%)$, SD: standard deviation $(\mathrm{cm} / \mathrm{s})$, Angle $\left({ }^{\circ}\right)$.

reflecting the number of parameters above the abnormality threshold (Figure 4).

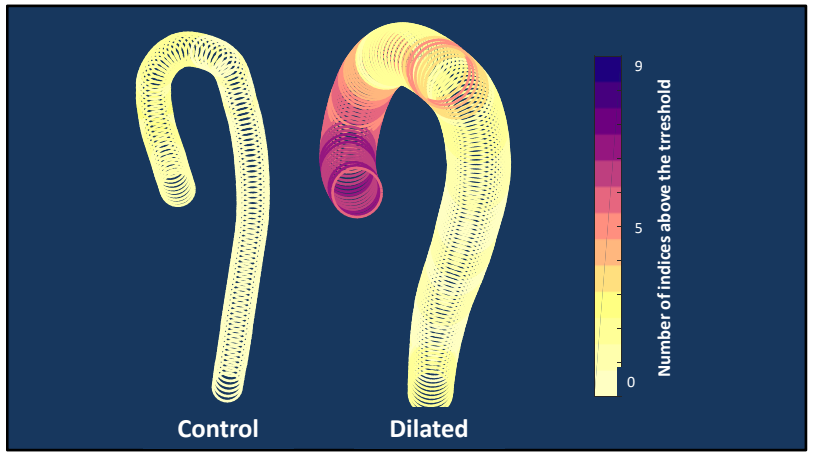

Figure 4. Flow indices mapping in a healthy control and a TAA patient.

\section{Conclusion}

This study uses 4D flow MRI to provide an insight into quantitative flow indices and their ability to characterize subsequent aortic alterations with aging and disease. Although variation of such flow indices, specifically backward flow, with disease is substantially higher than variations in diameters their full clinical value in dissection prediction needs to be demonstrated in larger longitudinal studies.

\section{Acknowledgments}

We would like to acknowledge the ECOS-SUD project number A15S04 (France-Argentina), ESME Sudria and the FRM project ING20150532487.

\section{References}

[1] Larson EW, Edwards WD. Risk factors for aortic dissection: a necropsy study of 161 cases. Am J Cardiol 1984; 53: 849-855.

[2] Kim JB, Spotnitz M, Lindsay ME, et al. Risk of Aortic Dissection in the Moderately Dilated Ascending Aorta. J Am Coll Cardiol 2016; 68: 12091219.

[3] Pape LA, Tsai TT, Isselbacher EM, et al. Aortic diameter $>$ or $=5.5 \mathrm{~cm}$ is not a good predictor of type A aortic dissection: observations from the International Registry of Acute Aortic Dissection (IRAD). Circulation 2007; 116: 1120-1127.

[4] Köhler B, Born S, van Pelt RF, et al. A Survey of Cardiac 4D PC-MRI Data Processing. In: Computer Graphics Forum. Wiley Online Library, http://onlinelibrary.wiley.com/doi/10.1111/cgf.12803/ full (2016, accessed 5 December 2016).

[5] Dyverfeldt P, Bissell M, Barker AJ, et al. 4D flow cardiovascular magnetic resonance consensus statement. J Cardiovasc Magn Reson 2015; 17: 72.

[6] Dietenbeck T, Craiem D, Rosenbaum D, et al. 3D aortic morphology and stiffness in MRI using semiautomated cylindrical active surface provides optimized description of the vascular effects of aging and hypertension. Comput Biol Med 2018; 103: 101108.

[7] Sigovan M, Hope MD, Dyverfeldt P, et al. Comparison of four-dimensional flow parameters for quantification of flow eccentricity in the ascending aorta. J Magn Reson Imaging JMRI 2011; 34: 12261230.

Address for correspondence:

Sophia Houriez--Gombaud-Saintonge.

15 rue de l'école de médecine 75006, Paris

sophia.houriez--gombaud-saintonge@etu.upmc.fr 